\title{
A CERTAIN NON-SINGULAR SYSTEM OF LENGTH THREE EQUATIONS OVER A GROUP
}

\author{
by S. WRETH* \\ (Received 6th October 1995)
}

\begin{abstract}
The Kervaire Conjecture is correct if it can be shown to hold for non-singular systems of equations of length 3. In this paper we prove it for the case of equations over a group $G$ where each equation has the form $a x b x^{-1} c y=1$ for $a, b, c \in G$.
\end{abstract}

1991 Mathematics subject classification: $20 \mathrm{~F} 05$.

\section{Introduction}

This paper deals with the conjecture of Kervaire that any non-singular system of equations has a solution over a group $G$.

Let $G$ be a group, $F$ the free group generated by $x_{1}, \ldots x_{n}$ and $W_{i} \in G * F$ for $i \in\{1, \ldots, m\}$. A system $\Sigma$ of $m$ equations $W_{i}=1$ in $n$ unknowns $x_{j}$ determines an $(m \times n)$-matrix whose $(i, j)$-entry is the exponent sum on $x_{j}$ in the word $W_{i}$, and we call the system $\Sigma$ non-singular if its exponent sum matrix has rank $m$. An infinite system of equations is called non-singular if every finite subsystem is non-singular.

The Kervaire Conjecture states that a non-singular system of equations over a group $G$ has a solution in some overgroup of $G$, i.e., that there exists a group $H$ and a homomorphism $\phi: G * F \rightarrow H$ such that $\phi(G)$ is an embedding and $\phi\left(W_{i}\right)=1$ for all $i$.

It has been shown by Gerstenhaber and Rothaus in [4] and [9] that this conjecture is true if $G$ is locally residually finite, and by Howie in [5] if $G$ is locally indicable. By focusing on the system $\Sigma$ rather than the group $G$ it has been shown in [6] and [7] that $\Sigma$ has a solution for $m \leq 2$ provided the length of the equations is at most 3 , where the length of an equation is the number of occurrences of unknowns. On the other hand it follows from results of Gersten in [3] that to prove the Kervaire Conjecture it is sufficient to study systems of equations of length 3 .

We shall prove the following result:

Theorem 1.1. Let $\Sigma$ be a non-singular system of equations in unknowns from a set $X$ over a group $G$, where each equation has the form

$$
a x b x^{-1} c y=1
$$

- This work is part of the author's PhD thesis. 
for $x, y \in X$ and $a, b, c \in G$. Then $\Sigma$ has a solution over $G$.

In Section 2 we describe our main tool, the weight test, and reduce the problem to three cases which are studied in Sections 3-5.

Some of the technical details have been omitted, but they can be found in [11].

I am very grateful to Jim Howie for several useful conversations and for reading a draft version of this paper.

\section{Preliminaries}

Our main tool is the weight test as given by Bogley and Pride in [1], and to explain this we first need to define the star graph of $\Sigma$ : The star graph of $\Sigma$ is the graph with one vertex for each $x_{i}$ and each $x_{i}^{-1}$ where $i \in\{1, \ldots, n\}$ and an edge labelled $g$ with initial vertex $x_{i}^{-\epsilon}$ and terminal vertex $x_{j}^{\delta}$ for every cyclic permutation of an equation that begins with $x_{i}^{\epsilon} g x_{j}^{\delta}$.

A weight function $\omega$ on $\Sigma$ is a real valued function defined on the edges of the star graph such that for each equation of length $n$ the sum of the weights of the $n$ edges corresponding to the coefficients in the equation is at most $n-2$. As indicated by Howie in [7] the weight test can be modified to state that if there exists a weight function $\omega$ on $\Sigma$ such that the weight of every non-empty, cyclically reduced path that represents the identity element of $G$ in the star graph of $\Sigma$ has weight at least 2 and every closed path has weight at least 0 , then $\Sigma$ has a solution in an overgroup of $G$.

The important point about the weight test is that it is not necessary to study all the relators in $G$. Instead it is sufficient to look at those relators that correspond to reduced, closed paths in the star graph. These paths are referred to as admissible paths.

The next step is to simplify the problem and give the layout (and some definitions) for the remains of the paper:

A standard argument shows that we may assume $\Sigma$ to be finite.

Let $\Gamma$ be the graph with one vertex for each element of $X$ and one edge for each equation in $\Sigma$, where the edge corresponding to Equation 1 has initial vertex $x$ and terminal vertex $y$.

We may assume that $\Gamma$ is connected: suppose $\Gamma$ consists of two components, then we can divide $X$ into two disjoint subsets $X_{1}$ and $X_{2}$, and $\Sigma$ into sets $\Sigma_{1}$ and $\Sigma_{2}$ of equations over $X_{1}$ and $X_{2}$. If $\Sigma_{1}$ and $\Sigma_{2}$ have solutions over $G$ in groups $H_{1}$ and $H_{2}$ respectively, then $\Sigma$ has a solution in $H_{1} *_{G} H_{2}$.

No vertex of $\Gamma$ can be the terminal vertex of more than one edge: if a vertex of $\Gamma$ were the terminal vertex of more than one edge, then $\Sigma$ would contain two equations of the form

$$
\begin{aligned}
a x b x^{-1} c y & =1 \\
d z e z^{-1} f y & =1
\end{aligned}
$$


where $x, y, z \in X$ and $a, b, c, d, e, f \in G$, and this contradicts our assumption that $\Sigma$ is non-singular.

If a vertex occurs as a terminal but not as an initial vertex in $\Gamma$, then one unknown occurs only once in $\Sigma$, and the equation with this one occurrence can be eliminated from the system, leading to a smaller system $\Sigma^{\prime}$ which is equivalent to $\Sigma$. Hence we may assume that each vertex of $\Gamma$ is the initial vertex of precisely one edge and the terminal vertex of precisely one edge, i.e., $\Gamma$ is a directed cycle.

If we number the elements of $X$ in the direction of this cycle, then $X=\left\{x_{1}, \ldots, x_{n}\right\}$ and $\Sigma$ has the form

$$
\begin{gathered}
a_{1} x_{1} b_{1} x_{1}^{-1} c_{1} x_{2}=1 \\
a_{2} x_{2} b_{2} x_{2}^{-1} c_{2} x_{3}=1 \\
\vdots \\
a_{n} x_{n} b_{n} x_{n}^{-1} c_{n} x_{1}=1
\end{gathered}
$$

where $a_{i}, b_{i}, c_{i} \in G$, and the star graph of $\Sigma$ is shown in Figure 2.1.

It is easy to see that we may assume $G$ to be generated by those elements of $G$ that actually appear in $\Sigma$ : Let $G^{\prime}$ be the subgroup of $G$ generated by the elements of $G$ that appear in $\Sigma$. Then $\Sigma$ has a solution in a group $H^{\prime}$ if and only if $\Sigma$ has a solution in $H^{\prime} *_{G} G$.

It was shown in [6] that we may assume that the edges of a maximal tree in the star graph of $\Sigma$ are labelled by the identity element of $G$, and since the star graph of $\Sigma$ contains a maximal tree with edges $a_{1}, \ldots, a_{n}, c_{2}, \ldots, c_{n}$ we shall assume that $a_{1}=a_{2}=\ldots=a_{n}=1$ and $c_{2}=c_{3}=\ldots=c_{n}=1$, so $G$ is generated by $c_{1}, b_{1}, \ldots, b_{n}$. If $b_{i}=1$ for some $i \in\{1, \ldots, n\}$ then $\Sigma$ has a solution in $G$, so we may assume that this is not the case.

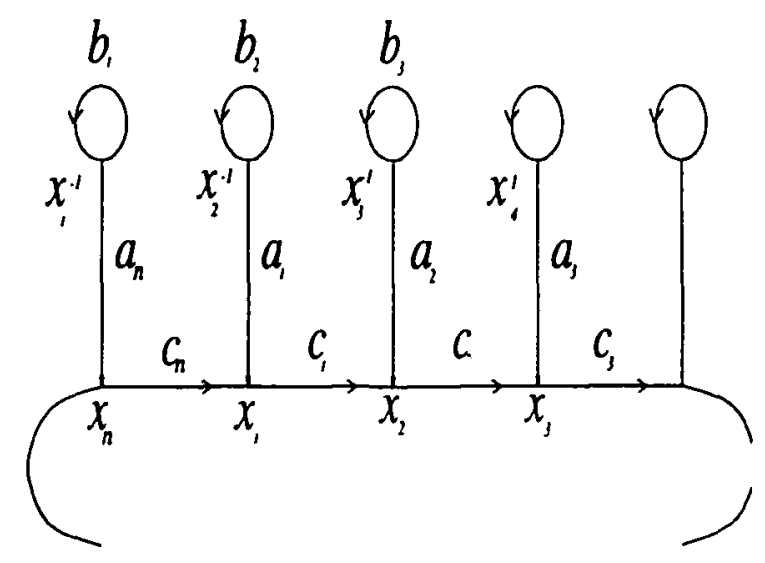

Figure 2.1: the star graph of $\Sigma$. 
We shall be using different techniques to show that $\Sigma$ has a solution over $G$ : Our first attempt will always be the weight test, i.e., a weight function $\omega$ for the edges of the star graph of $\Sigma$ such that $\omega\left(a_{i}\right)+\omega\left(b_{i}\right)+\omega\left(c_{i}\right) \leq 1$ for all $i$ and every admissible path has weight at least 2. If this fails (which is often the case when one or several $b_{i}$ have small order) we shall try to show that by adding the relator corresponding to an admissible path of weight less than 2 to a presentation of $G$, the group becomes residually finite. It then follows from the theorem of Gerstenhaber and Rothaus ([4]) that $\Sigma$ has a solution over $G$.

In most cases we will do this by showing that $G$ is in fact finite, and for this purpose we shall be using some well known results, summarised in the following lemma:

Lemma 2.1. The groups with the following presentations are finite:

- $\left\langle x, y ; x^{p}, y^{q},(x y)^{r}\right\rangle$ for $1 / p+1 / q+1 / r>1$

- $\left\langle x, y ; x^{3}, y^{2}, x^{-1} y(x y)^{m}\right\rangle$ where $m \in\{2,3,4\}$

- $\left\langle x, y, x^{3}, y^{2},\left(x^{-1} y\right)^{2}(x y)^{m}\right\rangle$ where $m \in\{2,3\}$

- $\left\langle x, y ; x^{3}, y^{2},\left(x^{-1} y x y\right)^{2}(x y)^{m}\right\rangle$ where $m \in\{0,1,2\}$

- $\left\langle x, y ; x^{3}, y^{m},\left(x y^{ \pm 1}\right)^{2}\right\rangle$ where $m \in\{3,4,5\}$

- $\left\langle x, y ; x^{3}, y^{m}, x y x y^{-1}\right\rangle$ where $m \in\{3,4,5\}$

- $\left\langle x, y ; x^{3}, y^{m}, x y x^{-1} y\right\rangle$ where $m \in\{3,4,5\}$

- $\left(x, y ; x^{3}, y^{m}, x y x^{-1} y^{-1}\right)$ where $m \in\{3,4,5\}$

- $\left\langle x, y ; x^{4}, y^{2} x^{-1} y(x y)^{m}\right\rangle$ where $m \in\{1,2\}$

- $\left\langle x, y ; x^{5}, y^{2}, x^{-1} y(x y)^{2}\right\rangle$

- $\left\langle x, y, z ; x^{3}, y^{2}, z^{2},[y, z],(x y)^{2},(x z)^{2}\right\rangle$

- $\left\langle x, y, z ; x^{3}, y^{2}, z^{2},[y, z],(x y)^{2},[x, z]\right)$

- $\left\langle x, y, z ; x^{3}, y^{2}, z^{2},[y, z],[x, y],[x, z]\right)$

- $\left\langle x, y, z ; x^{3}, y^{2}, z^{2},[y, z],[x, y],(x z)^{2}\right\rangle$

Proof. The groups in the first case are finite triangle groups. If - in the 2-generator cases $-x$ has order 3 and $y$ has order 2, these groups were shown to be finite in [2], if $x$ and $y$ both have order 3 they were shown to be finite in [8], and in the remaining cases GAP [10] was used to show finiteness.

We now introduce some notation that we will use throughout this paper: Let $K$ denote the set of indices $i$ such that

$$
a_{i} c_{i-1}^{-1} a_{i-1}^{-1} b_{i} a_{i-1} c_{i-1} a_{i}^{-1} \neq b_{i+1}^{ \pm 1} .
$$


The strategy of the proof of the theorem is to split the problem into five cases depending on the value of $|K|$. There are two cases which we can eliminate immediately; the first one deals with $|K|$ large:

Lemma 2.2 If $|K| \geq 4$ then $\Sigma$ has a solution over $G$.

Proof. We choose the following weights:

$$
\begin{gathered}
\omega\left(a_{i}\right)=\left\{\begin{array}{cc}
-1 / 2 & \text { for } i \in K \\
0 & \text { for } i \notin K
\end{array}\right. \\
\omega\left(b_{i}\right)=1 \text { for all } i \\
\omega\left(c_{i}\right)= \begin{cases}1 / 2 & \text { for } i \in K \\
0 & \text { for } i \notin K\end{cases}
\end{gathered}
$$

It is easy to convince oneself that if there is an admissible cycle with weight less than 2 , then there must be a relation of the form

$$
a_{i} c_{i-1}^{-1} a_{i-1}^{-1} b_{i} a_{i-1} c_{i-1} a_{i}^{-1} b_{i+1}^{ \pm 1}=1 .
$$

where $i \in K$, a contradiction.

The second case is the one where $K$ is the empty set:

Lemma 2.3. If $|K|=0$, then $\Sigma$ has a solution over $G$.

Proof. Since $|K|=0$ the relations

$$
c_{1}^{-1} b_{2} c_{1} b_{3}^{-1}=b_{3} b_{4}^{ \pm 1}=\ldots=b_{n} b_{1}^{ \pm 1}=b_{1} b_{2}^{ \pm 1}=1
$$

hold in $G$, so $G$ is generated by $c_{1}$ and $b_{1}$ and the relation $c_{1}^{-1} b_{1} c_{1} b_{1}^{ \pm 1}$ holds in $G$. This means that $G$ is metacyclic and hence residually finite, and it follows from the theorem of Gerstenhaber and Rothaus in [4] that $\Sigma$ has a solution over $G$.

We shall study the cases where $|K| \in\{1,2\}$ with the help of the following lemma:

Lemma 2.4. If none of the following is a relator in $G$, then $\Sigma$ has a solution over $G$ :

(1) $b_{i}^{2}$

(2) $a_{i} c_{i} \ldots c_{n} c_{1} \ldots c_{i-1} a_{i}^{-1} b_{i+1}^{ \pm 1}$ for $n=3$ (that is one of $a_{1} c_{1} c_{2} c_{3} a_{1}^{-1} b_{2}^{ \pm 1}, a_{2} c_{2} c_{3} c_{1} a_{2}^{-1} b_{3}^{ \pm 1}$ and $\left.a_{3} c_{3} c_{1} c_{2} a_{3}^{-1} b_{1}^{ \pm 1}\right)$

(3) $c_{1} c_{2} \ldots c_{n}$. 
Proof. The proof is a straightforward application of the weight test if we choose weights $\omega\left(a_{i}\right)=0, \omega\left(b_{i}\right)=2 / 3$ and $\omega\left(c_{i}\right)=1 / 3$.

The remaining sections of this paper will be devoted to the cases where $|K|$ is 1,2 and 3 respectively, but before we begin we introduce some more notation. We shall refer to reduced cycles with weight less than 2 as critical if their label could be a relator in $G$, i.e., if this does not contradict any previous assumptions.

When looking for reduced cycles in the star graph of $\Sigma$ that are critical we will encounter cycles that have weight less than 1. Although these cycles are not critical themselves, tracing their path twice (or more often) will lead to a critical cycle. If for instance $1 \in K$ then $a_{1} c_{n}^{-1} a_{n}^{-1} b_{1} a_{n} c_{n} a_{n}^{-1} b_{2}^{ \pm 1}$ is not a critical path but if it has weight less than 1 , then the cycle $\left(a_{1} c_{n}^{-1} a_{n}^{-1} b_{1} a_{n} c_{n} a_{n}^{-1} b_{2}^{ \pm 1}\right)^{2}$ may well be critical. In this case the cycles

$$
a_{1} c_{n}^{-1} a_{n}^{-1} b_{1} a_{n} c_{n} a_{n}^{-1} b_{2} a_{1} c_{n}^{-1} a_{n}^{-1} b_{1} a_{n} c_{n} a_{n}^{-1} b_{2}^{-1}
$$

and

$$
a_{1} c_{n}^{-1} a_{n}^{-1} b_{1} a_{n} c_{n} a_{n}^{-1} b_{2} a_{1} c_{n}^{-1} a_{n}^{-1} b_{1}^{-1} a_{n} c_{n} a_{n}^{-1} b_{2}^{-1}
$$

are also critical. In order to avoid having to write down these critical cycles every time they occur we shall refer to them as the critical cycles induced by a cycle (in this case by the cycle $a_{1} c_{n}^{-1} a_{n}^{-1} b_{1} a_{n} c_{n} a_{n}^{-1} b_{2}$ ) and list them only in terms of generators of $G$. So in this example we would list $\left(b_{1} b_{2}^{ \pm 1}\right)^{2}, b_{1} b_{2} b_{1} b_{2}^{-1}$ and $b_{1} b_{2} b_{1}^{-1} b_{2}^{-1}$.

Suppose there exists a critical cycle that contains $b_{i}$, then there exists another critical cycle that differs from the first one only in that $b_{i}$ is replaced by $b_{i}^{-1}$. If $b_{i}$ has order 2 in $G$, we shall ignore the second critical cycle, since it gives rise to the same relation in $G$ as the first one.

\section{3. $|\boldsymbol{K}|=\mathbf{1}$}

Throughout this section we shall assume that $K=\{1\}$, which means that $a_{1} c_{n}^{-1} a_{n}^{-1} b_{1} a_{n} c_{n} a_{1}^{-1} \neq b_{2}^{ \pm 1}$ and $a_{i} c_{i-1}^{-1} a_{i-1}^{-1} b_{i} a_{i-1} c_{i-1} a_{i}^{-1}=b_{i+1}^{ \pm 1}$ for all $i \neq 1$. Hence all $b_{i}$ will have the same order in $G$, and since we may assume that $a_{i}=1$ for all $i$ and $c_{i}=1$ for all $i \neq 1$ this implies that $c_{1}^{-1} b_{2} c_{1}=b_{3}^{ \pm 1}, b_{3}=b_{4}^{ \pm 1}, \ldots, b_{n}=b_{1}^{ \pm 1}$, so $G$ is generated by $b_{1}$ and $c_{1}$.

Lemma 3.1. The system $\Sigma$ has a solution over $G$ if $|K|=1$.

Proof. In each of the cases of Lemma 2.4 we shall either give weights that show that $\Sigma$ has a solution over $G$ or show that $G$ is residually finite, in which case it follows from the theorem of Gerstenhaber and Rothaus in [4] that $\Sigma$ has a solution over $G$.

We begin by assuming $b_{i}^{2}=1$ for all $i \in\{1,2, \ldots n\}$. Paths with label of the form 2 
and 3 in Lemma 2.4 will be dealt with below, so we shall assume that these are not admissible.

First we note that there is no admissible path using each $c_{i}$ exactly once: Since we have assumed that there are no relators of type 2 or 3 , such an admissible path would have to have at least two letters $b_{i}$ and $b_{j}$. Let us assume that there are exactly two such letters. Then

$$
b_{i} a_{i-1} c_{i-1} \ldots c_{j-2} a_{j-1}^{-1} b_{j}^{ \pm 1} a_{j-1} c_{j-1} \ldots c_{i-2} a_{i-1}^{-1}=1
$$

Since either

$$
a_{j-1}^{-1} b_{j}^{ \pm 1} a_{j-1} c_{j-1} \ldots c_{i-2} a_{i-1}^{-1} b_{i} a_{i-1}=c_{j-1} \ldots c_{i-2}
$$

or

$$
a_{i-1}^{-1} b_{i} a_{i-1} c_{i-1} \ldots c_{j-2} a_{j-1}^{-1} b_{j}^{ \pm 1} a_{j-1}=c_{i-1} \ldots c_{j-2}
$$

equation 2 implies that $c_{1} c_{2} \ldots c_{n}=1$, a contradiction. If there are more than two such letters, then there exist at least two letters $b_{i}$ and $b_{j}$ for which such a reduction can be performed, thus removing $a_{i-1}^{-1} b_{i} a_{i-1}$ and $a_{j-1}^{-1} b_{j} a_{j-1}$ from the equation and this process can be continued until we arrive at a relator of type 2 or 3 .

If $\left(c_{1} \ldots c_{n}\right)^{2}=1$ then $G$ is a dihedral group and contains a cyclic subgroup of finite index. Therefore $G$ is residually finite and $\Sigma$ has a solution over $G$. If $c_{1} \ldots c_{n}$ has infinite order we choose the following weights:

$$
\omega\left(a_{i}\right)=0=\omega\left(c_{i}\right) \text { and } \omega\left(b_{i}\right)=1 .
$$

Then the only reduced cycle that does not have at least two occurrences of $b_{i}$ has the form $a_{i}\left(c_{1} \ldots c_{n} c_{1} \ldots c_{i-1}\right)^{k} a_{i}^{-1} b_{i+1}^{ \pm 1}$; but such a relator would make $G$ cyclic so we may invoke the theorem of Gerstenhaber and Rothaus in [4] to show that $r(t)$ has a solution over $G$.

All other reduced cycles have weight at least 2 , so $\Sigma$ has a solution over $G$. We may now assume that $\left(c_{1} \ldots c_{n}\right)^{m}=1$ where $2<m<\infty$, and we choose the following weights:

$$
\omega\left(a_{1}\right)=-2 / m, \omega\left(c_{1}\right)=2 / m, \omega\left(a_{i}\right)=0=\omega\left(c_{i}\right) \text { for } i \neq 1
$$

and

$$
\omega\left(b_{i}\right)=1 \text { for all } i
$$

Then $a_{1} c_{n}^{-1} \ldots c_{i}^{-1} a_{i}^{-1} b_{i+1} a_{i} c_{i} \ldots c_{n} a_{1}^{-1} b_{2}^{ \pm 1}$ is not an admissible path, as this would imply $a_{1} c_{n}^{-1} a_{n}^{-1} b_{1} a_{n} c_{n} a_{1}^{-1}=b_{2}^{ \pm 1}$, a contradiction.

Any reduced cycle with only one occurrence of $b_{i}$ that is an admissible path makes $G$ cyclic, so we shall assume that any admissible path contains at least two occurrences of $b_{i}$. Hence weight less than 2 can only occur in reduced cycles that contain $b_{2}$ and 
one or no occurrence of $c_{1}$, which means that the only admissible paths of weight less than 2 are words of length $l \cdot(3+n)$ where $1<l<6$ induced by the cycle $a_{1} c_{1} \ldots c_{n} a_{1}^{-1} b_{2}^{ \pm 1}$ which has weight $1-2 / m$. It is easy to check that $G$ is residually finite for each of $l \in\{2,3,4,5\}$.

We still need to check cases 2 and 3 of Lemma 2.4. Let $a_{1} c_{1} c_{2} c_{3} a_{1}^{-1} b_{2}^{ \pm 1}=1$. Then $c_{1}=b_{2}^{ \pm 1}$ so $G$ is a cyclic group, and similarly $a_{2} c_{2} c_{3} c_{1} a_{2}^{-1} b_{3}^{ \pm 1}=1$ and $a_{3} c_{3} c_{1} c_{2} a_{3}^{-1} b_{1}^{ \pm 1}=1$ also imply that $G$ is cyclic.

Now let $c_{1} c_{2} \ldots c_{n}=1$. Then $a_{1} c_{n}^{-1} a_{n}^{-1} b_{1} a_{n} c_{n} a_{1}^{-1}=b_{2}^{ \pm 1}$, a contradiction. Hence it follows from Lemma 2.4 that $\Sigma$ has a solution over $G$.

\section{4. $|\boldsymbol{K}|=2$}

To simplify notation we shall assume that $K=\{1, j\}$, and hence $G$ is generated by $c_{1}, b_{2}$ and $b_{j+1}$. Case 1 of Lemma 2.4 is covered by the following four lemmas:

Lemma 4.1. Let $b_{i}^{2}=1$ for all $i$. Then $\Sigma$ has a solution over $G$.

Proof. If $c_{1} \ldots c_{n}=1$ then $G$ is a dihedral group, so we may assume that $c_{1} \ldots c_{n} \neq 1$ and choose the following weights:

$$
\begin{array}{r}
\omega\left(a_{i}\right)=\left\{\begin{array}{cc}
0 & \text { for } i \notin\{1, j\} \\
-1 / 2 & \text { for } i \in\{1, j\}
\end{array}\right. \\
\omega\left(b_{i}\right)=1 \text { for all } i
\end{array}
$$

Since $b_{i}=b_{i}^{-1}$ for all $i$, there are only three critical cycles: $a_{1} c_{1} \ldots c_{n} a_{1}^{-1} b_{2}$, $a_{j} c_{j} \ldots c_{n} c_{1} \ldots c_{j-1} a_{j}^{-1} b_{j+1}$ and $a_{1} c_{1} \ldots c_{j-1} a_{j}^{-1} b_{j+1} a_{j} c_{j} \ldots c_{n} a_{1}^{-1} b_{2}$. In the first case $c_{1} b_{2}=1$, in the second case $c_{1} b_{j+1}=1$ and in the last case $c_{1} b_{j+1} b_{2}=1$, so in either case $G$ is dihedral.

Lemma 4.2. Let $b_{i}^{2}=1$ for some $i \in\{1, \ldots n\}$ and $c_{1} \ldots c_{n}=1$. Then $\Sigma$ has a solution over $G$.

Proof. On account of Lemma 4.1 and for reasons of symmetry we may assume that $b_{2}^{2}=1$ and $b_{j+1}^{2} \neq 1$. $G$ is generated by $b_{2}$ and $b_{j+1}$, and furthermore $b_{i}^{2}=1$ if and only if $1<i \leq j$ and $a_{i} c_{i} \ldots c_{n} c_{1} \ldots c_{i-1} a_{i}^{-1} b_{i+1}^{ \pm 1} \neq 1$ for all $i$. Also, if $a_{1} c_{1} \ldots c_{i-1} a_{i}^{-1} b_{i+1} a_{i} c_{i} \ldots$ $c_{j-1} a_{j}^{-1} b_{j+1} a_{j} c_{j} \ldots c_{n} a_{1}^{-1} b_{2}$ is an admissible path then $G$ is cyclic, so we may assume that there are so such admissible paths. Since $n \geq 3$ we know that $j \neq 2$ or $j \neq n$, and in both cases we can find a weight function that has no critical paths.

Lemma 4.3. Let $c_{1} \ldots c_{n} \neq 1$ and $a_{i} c_{i} \ldots c_{n} c_{1} \ldots c_{i-1} a_{i}^{-1} b_{i+1}^{ \pm 1} \neq 1$ for all $i$ and $b_{i}^{2}=1$ for some $i$. Then $\Sigma$ has a solution over $G$. 
Proof. We begin by choosing the same weights as in the proof of Lemma 4.1. In this case there is only one critical cycle:

$$
a_{1} c_{1} \ldots c_{j+1} a_{j}^{-1} b_{j+1}^{\epsilon} a_{j} c_{j} \ldots c_{n} a_{1}^{-1} b_{2}^{\delta}, \text { where } \epsilon, \delta \in\{ \pm 1\}
$$

If this is a relator then $c_{1} b_{j+1}^{\epsilon} b_{2}^{\delta}=1$ for $\epsilon, \delta \in\{ \pm 1\}$, so $G$ is generated by any two of $c_{1}, b_{j+1}$ and $b_{2}$. If two of these have order 2 , then $G$ is dihedral. Consequently we may assume that exactly one of these has order 2 , so it must be either $b_{2}$ or $b_{j+1}$, since we had assumed that $b_{i}^{2}=1$ for some $i$.

Due to symmetry it is sufficient to study the case where $b_{2}^{2}=1$, and we choose the following weights:

$$
\begin{gathered}
\omega\left(a_{i}\right)= \begin{cases}1 / 3 & \text { for } j<i \leq n \\
0 & \text { for } 1 \leq i \leq j\end{cases} \\
\omega\left(b_{i}\right)= \begin{cases}1 & \text { for } 2 \leq i \leq j \\
2 / 3 & \text { else }\end{cases} \\
\omega\left(c_{i}\right)= \begin{cases}1 / 3 & \text { for } i=1 \\
0 & \text { for } i \neq 1\end{cases}
\end{gathered}
$$

With these weights there are no critical cycles provided $c_{1}$ has order at least 6 , since relators of the form

$$
a_{i}\left(c_{i} \ldots c_{n} c_{1} \ldots c_{i-1}\right)^{ \pm m} a_{i}^{-1} b_{i+1}^{ \pm 1} \text { and } a_{i} c_{i} \ldots c_{n} c_{1} \ldots c_{i-1} a_{i}^{-1} b_{i+1}^{ \pm m}
$$

make $G$ cyclic.

Let $c_{1}^{3}=1$. If $b_{j+1}$ has order at least 6 we choose the following weights:

$$
\begin{gathered}
\omega\left(a_{i}\right)= \begin{cases}2 / 3 & \text { for } j<i \leq n \\
0 & \text { for } 1 \leq i \leq j\end{cases} \\
\omega\left(b_{i}\right)= \begin{cases}1 & \text { for } 2 \leq i \leq j \\
1 / 3 & \text { else }\end{cases} \\
\omega\left(c_{i}\right)= \begin{cases}2 / 3 & \text { for } i=1 \\
0 & \text { for } i \neq 1\end{cases}
\end{gathered}
$$

and with these weights there are no critical cycles.

Let $b_{j+1}$ have order $m$ where $m \in\{3,4,5\}$. Then $\left\langle b_{2}, b_{j+1} ; b_{2}^{2}, b_{j+1}^{m}, W\right\rangle$ is a presentation for a homomorphic image of $G$ where $W$ is either $\left(b_{2} b_{j+1}\right)^{3}$ or $b_{2} b_{j+1}^{-1}\left(b_{2} b_{j+1}\right)^{2}$ (since $b_{2}$ has order 2), and all these groups are finite. Let $c_{1}$ have order 4 . If $b_{j+1}$ has order at least 4 choose 


$$
\begin{gathered}
\omega\left(a_{i}\right)= \begin{cases}1 / 2 & \text { for } j<i \leq n \\
0 & \text { for } 1 \leq i \leq j\end{cases} \\
\omega\left(b_{i}\right)= \begin{cases}1 & \text { for } 2 \leq i \leq j \\
1 / 2 & \text { else }\end{cases} \\
\omega\left(c_{i}\right)= \begin{cases}1 / 2 & \text { for } i=1 \\
0 & \text { for } i \neq 1\end{cases}
\end{gathered}
$$

so there are no critical cycles. Let $b_{j+1}^{3}=1$, then $G$ is the homomorphic image of a group with a presentation $\left\langle b_{2}, b_{j+1} ; b_{2}^{2}, b_{j+1}^{3}, W\right\rangle$ where $W$ is one of $\left(b_{2} b_{j+1}\right)^{4}$, $b_{2} b_{j+1}^{-1}\left(b_{2} b_{j+1}\right)^{3},\left(b_{2} b_{j+1}^{-1}\right)^{2}\left(b_{2} b_{j+1}\right)^{2}$ and $\left(b_{2} b_{j+1}^{-1} b_{2} b_{j+1}\right)^{2}$, and $G$ is finite in each of these cases.

Let $c_{1}$ have order 5 . If $b_{j+1}$ has order at least 4 choose the same weights as in the case where $c_{1}$ has order 4 to show that $\Sigma$ has a solution over $G$. If $b_{j+1}^{3}=1$ then $G$ is the homomorphic image of a group with a presentation $\left\langle b_{2}, b_{j+1} ; b_{2}^{2}, b_{j+1}^{3}, W\right\rangle$ where $W$ is one of $\left(b_{2} b_{j+1}\right)^{5}, b_{2} b_{j+1}^{-1}\left(b_{2} b_{j+1}\right)^{4},\left(b_{2} b_{j+1}^{-1}\right)^{2}\left(b_{2} b_{j+1}\right)^{3}$ and $\left(b_{2} b_{j+1}^{-1} b_{2} b_{j+1}\right)^{2} b_{2} b_{j+1}$, and $G$ is finite in each of these cases.

The strategy applied in the proof of Lemma 4.3 is one that we will be using throughout this paper: first we show that if one of the generators of $G$ has order no less than a certain (small) number then we can allocate weights in such a way that there are no critical cycles. Then we study each of the remaining cases where the order of this generator is small individually by allocating weights and showing that critical cycles induce relators that make $G$ finite.

Lemma 4.4. Let $b_{i}^{2}=1$ for some $i$ and $a_{l} c_{l} \ldots c_{n} c_{1} \ldots c_{l-1} a_{l}^{-1} b_{i+1}^{ \pm 1}=1$ for some $l$. Then $\Sigma$ has a solution over $G$.

Proof. We note that $c_{1} \ldots c_{n} \neq 1$ and that $G$ is generated by $b_{2}$ and $b_{j+1}$. As in the proof of Lemma 4.2 we may assume that $b_{2}^{2}=1$ and $b_{j+1}^{2} \neq 1$, but now there are two cases to be studied, depending on whether $b_{l+1}$ is conjugate to $b_{2}$ or to $b_{j+1}$.

In addition we may assume that $a_{1} c_{1} \ldots c_{j-1} a_{j}^{-1} b_{j+1}^{ \pm 1} a_{j} c_{j} \ldots c_{n} a_{1}^{-1} b_{2} \neq 1$, because this would imply $b_{j+1}=1$ or $b_{2}=1$, depending on whether $b_{l+1}$ is conjugate to $b_{2}$ or $b_{j+1}$. Let $b_{l+1}$ be conjugate to $b_{2}$; then $a_{i} c_{i} \ldots c_{n} c_{1} \ldots c_{i-1}^{-1} a_{i}^{-1} b_{i+1}^{ \pm 1}=1$ if and only if $1 \leq i<j$. If $a_{i} c_{i} \ldots c_{n} c_{1} \ldots c_{i-1} a_{i}^{-1} b_{i+1}^{ \pm m}=1$ or $a_{i}\left(c_{i} \ldots c_{n} c_{1} \ldots c_{i-1}\right)^{ \pm m} a_{i}^{-1} b_{i+1}=1$ for $j \leq i \leq n$ or $a_{i} c_{i} \ldots c_{j-1} a_{j}^{-1} b_{j+1}^{m} a_{j} c_{j-1}^{-1} \ldots c_{i}^{-1} a_{i}^{-1} b_{i+1}=1$ for $1 \leq i<j$ then $G$ is cyclic, so we may assume that these are not relators. If $\left(a_{i} c_{i} \ldots c_{n} c_{1} \ldots c_{i-1} a_{i}^{-1} b_{i+1}^{ \pm 1}\right)^{2}=1$ or

$$
a_{i} c_{i} \ldots c_{n} c_{1} \ldots c_{i-1} a_{i}^{-1} b_{i+1} a_{i} c_{i} \ldots c_{n} c_{1} \ldots c_{i-1} a_{i}^{-1} b_{i+1}^{-1}=1
$$

for $j \leq i \leq n$ then in $G$ we have $\left(b_{2} b_{j+1}\right)^{2}=1$ or $b_{2} b_{j+1} b_{2} b_{j+1}^{-1}=1$. In the first case $G$ is dihedral and in the second metacyclic, so we may assume that these are not relators either. Similarly relators of the form $a_{i} c_{i} \ldots c_{j-1} a_{j}^{-1} b_{j+1}^{m} a_{j} c_{j-1}^{-1} \ldots c_{i}^{-1} a_{i}^{-1} b_{i+1}=1$ would make $G$ metacyclic. 
Now let $b_{1+1}$ be conjugate to $b_{j+1}$; then $a_{i} c_{i} \ldots c_{n} c_{1} \ldots c_{i-1}^{-1} a_{i}^{-1} b_{i+1}^{ \pm 1}=1$ if and only if $j \leq i \leq n$. In addition we may assume that none of

$$
\begin{gathered}
a_{i} c_{i} \ldots c_{n} c_{1} \ldots c_{i-1} a_{i}^{-1} b_{i+1}^{ \pm m} \\
a_{i}\left(c_{i} \ldots c_{n} c_{1} \ldots c_{i-1}\right)^{ \pm m} a_{i}^{-1} b_{i+1} \\
\left(a_{i} c_{i} \ldots c_{n} c_{1} \ldots c_{i-1} a_{i}^{-1} b_{i+1}^{ \pm 1}\right)^{2} \\
a_{i} c_{i} \ldots c_{j-1} a_{j}^{-1} b_{j+1}^{m} a_{j} c_{j-1}^{-1} \ldots c_{i}^{-1} a_{i}^{-1} b_{i+1}
\end{gathered}
$$

and

$$
a_{i} c_{i} \ldots c_{n} c_{1} \ldots c_{i-1} a_{i}^{-1} b_{i+1} a_{i}\left(c_{i} \ldots c_{n} c_{1} \ldots c_{i-1}\right)^{-1} a_{i}^{-1} b_{i+1}
$$

is a relator in $G$ for $1 \leq i \leq j$.

In each of these two cases we can now use the approach described after the proof of Lemma 4.3: we allocate weights depending on the order of $b_{j+1}$ to show that if the order is at least 6 then there are no critical cycles and that if the order is either 3,4 or 5 then the relators induced by the critical cycles make $G$ into a finite group. We omit the details.

These four lemmas show that $\Sigma$ has a solution over $G$ if $b_{i}^{2}=1$ for some $i \in\{1, \ldots, n\}$ and we now turn to the case where $b_{i}^{2} \neq 1$ for all $i \in\{1, \ldots, n\}$ :

Lemma 4.5. Let $b_{i}^{2} \neq 1$ for all $i \in\{1, \ldots, n\}$. Then $\Sigma$ has a solution over $G$.

Proof. On account of Lemma 2.4 it is sufficient to study the cases where one of $a_{1} c_{i} \ldots c_{n} c_{1} \ldots c_{i-1} a_{i}^{-1} b_{i+1}^{ \pm 1}$ for $n=3$ or $c_{1} \ldots c_{n}$ is a relator in $G$. Let $a_{1} c_{1} c_{2} c_{3} a_{1}^{-1} b_{2}^{ \pm 1}=1$. If $j=3$ then $a_{2} c_{2} c_{3} c_{1} a_{2}^{-1} b_{3}^{ \pm 1}=1$ and neither $a_{3} c_{3} c_{1} c_{2} a_{3}^{-1} b_{1}^{ \pm 1}$ nor $c_{1} c_{2} c_{3}$ is a relator in $G$; if $j=2$ then $a_{2} c_{2} c_{3} c_{1} a_{2}^{-1} b_{3}^{ \pm 1}$ is not a relator either and we choose:

$$
\begin{gathered}
\omega\left(a_{i}\right)=\left\{\begin{array}{cl}
-1 / 3 & \text { for } i=j \\
0 & \text { else }
\end{array}\right. \\
\omega\left(b_{i}\right)=2 / 3 \text { for all } i \\
\omega\left(c_{i}\right)= \begin{cases}2 / 3 & \text { for } i=j \\
1 / 3 & \text { else }\end{cases}
\end{gathered}
$$

Again there are no critical cycles. A similar weight function can be used to show that there are no critical cycles if $a_{2} c_{2} c_{3} c_{1} a_{2}^{-1} b_{3}^{ \pm 1}=1$ or $a_{3} c_{3} c_{1} c_{2} a_{3}^{-1} b_{1}^{ \pm 1}=1$.

Now let $c_{1} \ldots c_{n}=1$ Then $G$ is generated by $b_{2}$ and $b_{j+1}$ and there are no relators of the form $a_{i} c_{i} \ldots c_{n} c_{1} \ldots c_{i-1} a_{i}^{-1} b_{i+1}^{ \pm 1}$. In addition we may assume that neither

$$
a_{1} c_{1} \ldots c_{j-1} a_{j}^{-1} b_{j+1}^{m} a_{j} c_{j-1}^{-1} \ldots c_{1}^{-1} a_{1}^{-1} b_{2}^{ \pm 1}
$$


nor

$$
a_{1} c_{1} \ldots c_{j-1} a_{j}^{-1} b_{j+1} a_{j} c_{j-1}^{-1} \ldots c_{1}^{-1} a_{1}^{-1} b_{2}^{ \pm m}
$$

is a relator, since this would make $G$ cyclic.

Applying the same principles as in Lemma 4.3 we can allocate weights depending on the order of $b_{2}$ and show that existing critical cycles induce relators that make $G$ finite. Again we omit the details, and this completes the proof of the lemma.

In the section we have proved the following lemma:

Lemma 4.6. The system $\Sigma$ has a solution over $G$ if $|K|=2$.

5. $|\boldsymbol{K}|=\mathbf{3}$

Throughout this section we shall assume that $|K|=3$, and for notational convenience we choose $K=\{1, j, k\}$ where $1<j<k$. In this connection the letters $j$ and $k$ will be meant to be fixed, whereas the letters $i$ and $l$ will stand for variables.

In this section we shall not be using Lemma 2.4 , but shall organise our results as follows: First we show that $\Sigma$ has a solution if no $b_{i}$ has order 2 and in the next two lemmas we show that $\Sigma$ has a solution if $c_{1} \ldots c_{n} \neq 1$. Then we study the case where all $b_{i}$ have order 2 , and the last two lemmas are devoted to the remaining case when some but not all $b_{i}$ have order 2 and $c_{1} \ldots c_{n}=1$.

For $|K|=3$ it is particularly easy to show that $\Sigma$ has a solution over $G$ if $b_{i}^{2} \neq 1$ for all $i$, so we begin by doing this:

Lemma 5.1. Let $b_{i}^{2} \neq 1$ for all $i \in\{1, \ldots n\}$. Then $\Sigma$ has a solution over $G$.

Proof. We choose the following weights:

$$
\begin{gathered}
\omega\left(a_{i}\right)=\left\{\begin{array}{cc}
-1 / 3 & \text { for } i \in\{1, j, k\} \\
0 & \text { for } i \notin\{1, j, k\}
\end{array}\right. \\
\omega\left(b_{i}\right)=2 / 3 \text { for all } i \\
\omega\left(c_{i}\right)= \begin{cases}2 / 3 & \text { for } i \in\{1, j, k\} \\
1 / 3 & \text { for } i \notin 1, j, k\}\end{cases}
\end{gathered}
$$

There are no critical cycles, so this proves the lemma.

From now on we shall assume that there exists at least one relation of the form $b_{i}^{2}=1$. Our next step will be to show that $\Sigma$ has a solution over $G$ if $c_{1} \ldots c_{n} \neq 1$. We shall prove this with the help of two lemmas, and we begin with a useful observation: 
Reduced cycles of the form

$$
\begin{aligned}
a_{1} c_{n}^{-1} \ldots c_{l}^{-1} a_{l}^{-1} b_{l+1} a_{l} c_{l} \ldots c_{n} a_{1}^{-1} b_{2}^{ \pm 1} & \text { for } k \leq l \leq n \\
a_{k} c_{k-1}^{-1} \ldots c_{l}^{-1} a_{l}^{-1} b_{l+1} a_{l} c_{l} \ldots c_{k-1} a_{k}^{-1} b_{k+1}^{ \pm 1} & \text { for } j \leq l<k \\
a_{j} c_{j-1}^{-1} \ldots c_{l}^{-1} a_{l}^{-1} b_{l+1} a_{l} c_{l} \ldots c_{j-1} a_{j}^{-1} b_{j+1}^{ \pm 1} & \text { for } 1 \leq l<j
\end{aligned}
$$

cannot be admissible, as they would imply the existence of a relator of the form

$$
a_{i} c_{i-1}^{-1} a_{i-1}^{-1} b_{i} a_{i-1} c_{i-1} a_{i}^{-1} b_{i+1}^{ \pm 1} \text { for } i \in K
$$

a contradiction.

Lemma 5.2. Let there be no relation of the form $a_{i} c_{i} \ldots c_{n} c_{1} \ldots c_{i-1} a_{i}^{-1} b_{i+1}^{ \pm 1}=1$ and let $c_{1} \ldots c_{n} \neq 1$ in $G$. Then $\Sigma$ has a solution over $G$.

Proof. We begin by choosing the following weights:

$$
\begin{gathered}
\omega\left(a_{1}\right)=\omega\left(a_{j}\right)=\omega\left(a_{k}\right)=-1 / 3, \omega\left(a_{1}\right)=0 \text { for } i \neq 1, j, k \\
\omega\left(b_{i}\right)=1 \text { for all } i \\
\omega\left(c_{1}\right)=\omega\left(c_{j}\right)=\omega\left(c_{k}\right)=1 / 3, \omega\left(c_{i}\right)=0 \text { for } i \neq 1, j, k .
\end{gathered}
$$

Cycles of the form $a_{i} c_{i} \ldots c_{l-1} a_{l} b_{l+1} a_{1}^{-1} c_{l-1}^{-1} \ldots c_{i}^{-1} a_{i}^{-1} b_{i+1}^{ \pm 1}$ can have weight less than 2 only if $l \in K$ and $i+1, \ldots, l-1 \notin K$. But then the cycle cannot be admissible, as this would imply $a_{1} c_{l-1}^{-1} a_{l-1}^{-1} b_{l} a_{l-1} c_{l-1} a_{l}^{-1} b_{l+1}^{ \pm 1}=1$, a contradiction.

Hence there are three critical cycles:

$$
\begin{gathered}
a_{1} c_{1} \ldots c_{j-1} a_{j}^{-1} b_{j+1}^{ \pm 1} a_{j} c_{j} \ldots c_{n} a_{1}^{-1} b_{2} \\
a_{1} c_{1} \ldots c_{k-1} a_{k}^{-1} b_{k+1}^{ \pm 1} a_{k} c_{k} \ldots c_{n} a_{1}^{-1} b_{2} \\
a_{j} c_{j} \ldots c_{k-1} a_{k}^{-1} b_{k+1}^{ \pm 1} a_{k} c_{k} \ldots c_{j-1} a_{j}^{-1} b_{j+1}
\end{gathered}
$$

No two of these cycles can be admissible at the same time: assume for example that the first two are; then

$$
a_{j}^{-1} b_{j+1}^{ \pm 1} a_{j} c_{j} \ldots c_{k-1}=c_{j} \ldots c_{k-1} a_{k}^{-1} b_{k+1}^{ \pm 1} a_{k}
$$

But this implies $a_{k} c_{k-1}^{-1} a_{k-1}^{-1} b_{k} a_{k-1} c_{k-1} a_{k}^{-1} b_{k+1}^{ \pm 1}=1$, a contradiction.

So we may assume (by symmetry) that only the word in (3) is a relator, and we choose the following weights: 


$$
\begin{gathered}
\omega\left(a_{1}\right)=-1 / 2=\omega\left(a_{k}\right), \omega\left(a_{i}\right)=0 \text { for } i \neq 1, k \\
\omega\left(b_{i}\right)=1 \text { for all } i \\
\omega\left(c_{1}\right)=1 / 2=\omega\left(c_{k}\right), \omega\left(c_{i}\right)=0 \text { for } i \neq 1, k .
\end{gathered}
$$

Now the only critical cycle has label

$$
a_{k} c_{k-1}^{-1} \ldots c_{l}^{-1} a_{l}^{-1} b_{l+1} a_{l} c_{l} \ldots c_{k-1} a_{k}^{-1} b_{k+1}^{ \pm 1} \text { for } 1 \leq l<j
$$

If this is an admissible cycle then $G$ is generated by $b_{2}$ and $b_{j+1}$ and if $b_{2}$ and $b_{j+1}$ both have order 2 then $G$ is a dihedral group, so we may assume that this is not the case.

Let $b_{2}$ have order greater than 2 . Since $b_{2}$ has the same order as $b_{k+1}$, it follows that $b_{k+1}$ also has order greater than 2 .

We choose the following weights:

$$
\begin{gathered}
\omega\left(a_{i}\right)=\left\{\begin{array}{cl}
1 / 3 & \text { for } 1<i<j \text { or } k<i \leq n \\
-1 / 3 & \text { for } i=j \\
0 & \text { else }
\end{array}\right. \\
\omega\left(b_{i}\right)= \begin{cases}1 & \text { for } j+1 \leq i \leq k \\
2 / 3 & \text { else }\end{cases} \\
\omega\left(c_{i}\right)= \begin{cases}1 / 3 & \text { for } i=1 \\
2 / 3 & \text { for } i=j \\
0 & \text { for } i \notin\{1, j\}\end{cases}
\end{gathered}
$$

In either case there are no critical cycles, so we may assume that $b_{2}$ and $b_{k+1}$ have order 2 and $b_{j+1}$ has order greater than 2 . We choose the following weights:

$$
\begin{gathered}
\omega\left(a_{i}\right)=\left\{\begin{array}{cl}
1 / 3 & \text { for } j<i<k \\
0 & \text { for } 1 \leq i<j \text { or } k<i \leq n \\
-1 / 3 & \text { for } i \in\{j, k\}
\end{array}\right. \\
\omega\left(b_{i}\right)= \begin{cases}2 / 3 & \text { for } j<i \leq k \\
1 & \text { for } 1 \leq i \leq j \text { or } k<i \leq n\end{cases} \\
\omega\left(c_{i}\right)= \begin{cases}1 / 3 & \text { for } i=j \\
2 / 3 & \text { for } i=k \\
0 & \text { for } i \neq j, k\end{cases}
\end{gathered}
$$

The critical cycles have label

$$
a_{i} c_{i} \ldots c_{n} c_{1} \ldots c_{j-1} a_{i}^{-1} b_{j+1} a_{j} c_{j-1}^{-1} \ldots c_{1}^{-1} c_{n}^{-1} \ldots c_{i}^{-1} a_{i}^{-1} b_{i+1}^{ \pm 1}
$$


where $k \leq i \leq n$ and

$$
a_{i} c_{i} \ldots c_{n} c_{1} \ldots c_{j-1} a_{i}^{-1} b_{j+1}^{2} a_{j} c_{j-1}^{-1} \ldots c_{1}^{-1} c_{n}^{-1} \ldots c_{i}^{-1} a_{i}^{-1} b_{i+1}^{ \pm 1}
$$

where $k<i \leq n$. If the first one is an admissible path, then $b_{k+1}$ has the same order as $b_{j+1}$, a contradiction, so we may assume this not to be a relator.

If the second one is an admissible path then $b_{j+1}$ has order 4 . We also note that if $c_{1}$ has order less than 4 , then so does $b_{j+1} b_{2}$, since the relator in (3) implies $c_{1} b_{j+1}^{ \pm 1} b_{2}=1$ and $b_{2}$ has order 2 , so $G$ is a finite group. Hence we may assume that $c_{1}$ has order at least 4 , and we choose

$$
\begin{gathered}
\omega\left(a_{i}\right)= \begin{cases}1 / 2 & \text { for } j<i<k \\
0 & \text { else }\end{cases} \\
\omega\left(b_{i}\right)= \begin{cases}1 & \text { for } 1 \leq i \leq j \text { or } k<i \leq n \\
1 / 2 & \text { for } j<i \leq k\end{cases} \\
\omega\left(c_{i}\right)= \begin{cases}1 / 2 & \text { for } i=k \\
0 & \text { for } i \neq k\end{cases}
\end{gathered}
$$

with these weights there are no critical cycles.

Lemma 5.3. Let there be a relation of the form $a_{i} c_{i} \ldots c_{n} c_{1} \ldots c_{i-1} a_{i}^{-1} b_{i+1}^{ \pm 1}=1$ in $G$. Then $\Sigma$ has a solution over $G$.

Proof. Let $a_{1} c_{1} \ldots c_{n} a_{1}^{-1} b_{2}^{ \pm 1}=1$. Then $a_{i} c_{i} \ldots c_{n} c_{1} \ldots c_{i-1} a_{i}^{-1} b_{i+1}^{ \pm 1}=1$ for $1 \leq i<j$. If in addition $a_{i} c_{i} \ldots c_{n} c_{1} \ldots c_{i-1} a_{i}^{-1} b_{i+1}^{ \pm 1}=1$ for $j \leq i \leq n$ then it follows that $a_{i} c_{i-1}^{-1} a_{i-1}^{-1} b_{i} a_{i-1} c_{i-1} a_{i}^{-1} b_{i+1}^{ \pm 1}=1$ for $i \in K$, a contradiction. So we may assume that this is not the case.

Since $c_{1} \ldots c_{n}$ cannot be a relator either, we choose the following weights:

$$
\begin{aligned}
& \omega\left(a_{j}\right)=-1 / 2=\omega\left(a_{k}\right), \omega\left(a_{i}\right)=0 \text { for } i \neq j, k \\
& \omega\left(b_{i}\right)=1 \text { for all } i \\
& \omega\left(c_{j}\right)=1 / 2=\omega\left(c_{k}\right), \omega\left(c_{i}\right)=0 \text { for } i \neq j, k .
\end{aligned}
$$

There are two types of critical cycles:

$$
a_{i} c_{i} \ldots c_{n} c_{1} \ldots c_{j-1} a_{j}^{-1} b_{j+1} a_{j} c_{j-1}^{-1} \ldots c_{1}^{-1} c_{n}^{-1} \ldots c_{i}^{-1} a_{i}^{-1} b_{i+1}^{ \pm 1}
$$

where $k \leq i \leq n$, and

$$
a_{i} c_{i} \ldots c_{n} c_{1} \ldots c_{j-1} a_{j}^{-1} b_{j+1} a_{j} c_{j} \ldots c_{i-1} a_{i}^{-1} b_{i+1}^{ \pm 1}
$$


where $k \leq i<n$. If either of these is a relator then $G$ is generated by $b_{2}$ and $b_{j+1}$, so we may assume that $b_{2}$ and $b_{j+1}$ do not both have order 2 . In both cases a relator of the form

$$
a_{i} c_{i} \ldots c_{n} c_{1} \ldots c_{i-1} a_{i}^{-1} b_{i+1}^{ \pm m} \text { or } a_{i}\left(c_{i} \ldots c_{n} c_{1} \ldots c_{i-1}\right)^{ \pm m} a_{i}^{-1} b_{i+1}
$$

where $k \leq i \leq n$ would make $G$ cyclic, so we shall assume that these are not admissible paths. Similarly we shall assume that paths with label

$$
a_{i} c_{i-1}^{-1} \ldots c_{j}^{-1} a_{j}^{-1} b_{j+1} a_{j} c_{j} \ldots c_{i-1} a_{i}^{-1} b_{i+1}^{ \pm m}
$$

or

$$
a_{i} c_{i-1}^{-1} \ldots c_{j}^{-1} a_{j}^{-1} b_{j+1}^{ \pm m} a_{j} c_{j} \ldots c_{i-1} a_{i}^{-1} b_{i+1}
$$

are not admissible for $k \leq i \leq n$.

We begin with the case where

$$
a_{i} c_{i} \ldots c_{n} c_{1} \ldots c_{j-1} a_{j}^{-1} b_{j+1} a_{j} c_{j-1}^{-1} \ldots c_{1}^{-1} c_{n}^{-1} \ldots c_{i}^{-1} a_{i}^{-1} b_{i+1}^{ \pm 1}=1
$$

for $k \leq i \leq n$. Let $b_{j+1}$ have order greater than 2 ; then $b_{k+1}$ also has order greater than 2. We choose the following weights:

$$
\begin{gathered}
\omega\left(a_{i}\right)=\left\{\begin{array}{cl}
1 / 3 & \text { for } j<i<k \\
0 & \text { for } k \leq i \leq n \text { or } 1 \leq i<j \\
-1 / 3 & \text { for } i=j
\end{array}\right. \\
\omega\left(b_{i}\right)= \begin{cases}1 & \text { for } 2 \leq i \leq j \\
2 / 3 & \text { for } j<i \leq n\end{cases} \\
\omega\left(c_{i}\right)= \begin{cases}1 / 3 & \text { for } i=1 \text { or } i=j \text { or } k \leq i \leq n \\
0 & \text { for } 1<i<j \text { or } j+1 \leq i<k\end{cases}
\end{gathered}
$$

Then there are no critical cycles.

We may now assume that $b_{j+1}^{2}=1$ and that $b_{2}$ has order greater than 2. If $\left(a_{k} c_{k} \ldots c_{n} c_{1} \ldots c_{k+1} a_{k}^{-1} b_{k+1}^{ \pm 1}\right)^{2}$ is a relator, then we have $1=\left(c_{1} b_{k+1}^{ \pm 1}\right)^{2}=\left(b_{2} b_{j+1}^{ \pm 1}\right)^{2}$, so $G$ is dihedral. Similarly, if

$$
a_{k} c_{k} \ldots c_{n} c_{1} \ldots c_{k-1} a_{k}^{-1} b_{k+1} a_{k}\left(c_{k} \ldots c_{n} c_{1} \ldots c_{k-1}\right)^{-1} a_{k}^{-1} b_{k+1}
$$

is an admissible path then $G$ is the homomorphic image of a group with a presentation $\left\langle b_{2}, b_{j+1} ; b_{j+1}^{2},\left[b_{2}, b_{j+1}\right]\right\rangle$ and therefore $G$ is an abelian group. Hence we may assume that none of these is an admissible path.

As in the proof of Lemma 4.3 it is now possible to show that depending on the order 
of $b_{2}$ weights can be allocated in such a way that either there are no critical cycles or that the critical cycles induce relators that make $G$ finite.

This concludes the first part of the proof and accordingly we assume that

$$
a_{i} c_{i} \ldots c_{n} c_{1} \ldots c_{j-1} a_{j}^{-1} b_{j+1} a_{j} c_{j-1}^{-1} \ldots c_{1}^{-1} c_{n}^{-1} \ldots c_{i}^{-1} a_{i}^{-1} b_{i+1}^{ \pm 1} \neq 1
$$

and

$$
a_{i} c_{i} \ldots c_{n} c_{1} \ldots c_{j-1} a_{j}^{-1} b_{j+1} a_{j} c_{j} \ldots c_{i-1} a_{i}^{-1} b_{i+1}^{ \pm 1}=1
$$

for $k \leq i \leq n$.

If $b_{2}$ and $b_{k+1}$ both have order 2 , we may choose them as generators for $G$, in which case $G$ is the homomorphic image of a dihedral group. We begin by assuming that $b_{k+1}$ has order greater than 2 and for the sake of consistency we shall continue to assume that $G$ is generated by $b_{2}$ and $b_{j+1}$. We can proceed in a similar way as in Lemma 4.3 , by first allocating weights for the case where $b_{j+1}$ has order at least 6 in such a way that there are no critical cycles. If $b_{j+1}$ has order 4 or 5 and $b_{k+1}$ has order less than 4 then $G$ is the homomorphic image of a finite triangle group, so we may assume that $b_{k+1}$ has order at least 4 and we choose weights that avoid critical cycles. Next we let $b_{j+1}^{3}=1$. If $b_{k+1}$ has order less than 6 then $G$ is the homomorphic image of a finite triangle group, so we shall assume that $b_{k+1}$ has order at least 6 and choose weights that avoid critical cycles.

Now let $b_{j+1}^{2}=1$, so we may assume that $b_{2}$ and $b_{k+1}$ have order greater than 2 . Similar considerations depending on the order of $b_{2}$ show that $\Sigma$ has a solution over $G$, so we shall now assume that $b_{k+1}$ has order 2 . As mentioned before we may assume that neither $b_{j+1}$ nor $b_{2}$ has order 2 .

We can follow the same pattern as previously by studying several cases depending on the order of $b_{j+1}$, and noting that we may assume that there are no relators of the form $b_{2} b_{j+1}^{ \pm m}, b_{2} b_{k+1}^{ \pm m}, b_{j+1} b_{k+1}^{ \pm m}, b_{j+1} b_{2}^{ \pm m}, b_{k+1} b_{j+1}^{ \pm m}$ and $b_{k+1} b_{2}^{ \pm m}$, since they make $G$ cyclic.

This completes the proof of the lemma.

The results of the last two lemmas of this section are summarised in the following corollary:

Corollary 5.4. If $c_{1} \ldots c_{n} \neq 1$ then $\Sigma$ has a solution over $G$.

We now proceed to study the case where $c_{1} \ldots c_{n}$ is a relator in $G$; in this case $G$ is generated by $b_{2}, b_{j+1}$ and $b_{k+1}$, and we start with the simple case where all $b_{1}$ have order 2:

Lemma 5.5. Let $b_{i}^{2}=1$ for all $i$. Then $\Sigma$ has a solution over $G$.

Proof. On account of Corollary 5.4 we may assume that $c_{1} \ldots c_{n}=1$. This implies that there can be no relator of the form $a_{i} c_{i} \ldots c_{n} c_{1} \ldots c_{i-1} a_{i}^{-1} b_{i+1}$, and we allocate weights to the star graph of $\Sigma$ as shown below: 


$$
\begin{array}{r}
\omega\left(a_{i}\right)=\left\{\begin{array}{cc}
0 & \text { for } i \notin\{1, j, k\} \\
-2 / 3 & \text { for } i \in\{1, j, k\}
\end{array}\right. \\
\omega\left(b_{i}\right)=1 \text { for all } i \\
\omega\left(c_{i}\right)= \begin{cases}2 / 3 & \text { for } i \in\{1, j, k\} \\
0 & \text { for } i \notin\{1, j, k\}\end{cases}
\end{array}
$$

The only critical cycle has label

$$
a_{1} c_{1} \ldots c_{j-1} a_{j}^{-1} b_{j+1} a_{j} c_{j} \ldots c_{k-1} a_{k}^{-1} b_{k+1} a_{k} c_{k} \ldots c_{n} a_{1}^{-1} b_{2}
$$

If this is an admissible path then $b_{j+1} b_{k+1} b_{2}=1$, so $G$ is the homomorphic image of a dihedral group and $\Sigma$ has a solution over $G$.

In the next two lemmas we look at the case where some but not all $b_{i}$ have order 2.

Lemma 5.6. Let

$$
a_{1} c_{1} \ldots c_{j-1} a_{j}^{-1} b_{j+1}^{\eta} a_{j} c_{j} \ldots c_{k-1} a_{k}^{-1} b_{k+1}^{\epsilon} a_{k} c_{k} \ldots c_{n} a_{1}^{-1} b_{2}^{\delta}=1
$$

where each of $\epsilon, \delta$ and $\eta$ is either 1 or -1 . Then $\Sigma$ has a solution over $G$.

Proof. On account of Lemma 5.5 it is sufficient to study the case where not all $b_{i}$ have order 2 , and because of Corollary 5.4 we may also assume that $c_{1} \ldots c_{n}=1$. For reasons of symmetry it is sufficient to investigate the case where $b_{2}^{2} \neq 1$. Note that we may assume that $b_{2} b_{k+1}^{ \pm m}, b_{2} b_{j+1}^{ \pm m}, b_{2}^{ \pm m} b_{j+1}, b_{j+1} b_{k+1}^{ \pm m}, b_{2}^{ \pm m} b_{k+1}$ and $b_{k+1} b_{j+1}^{ \pm m}$ are not relators, since in conjunction with the relation $b_{j+1}^{\eta} b_{k+1}^{\epsilon+1} b_{2}^{\delta}=1$ this would turn $G$ into a cyclic group.

We may also assume that $G$ is generated by $b_{j+1}$ and $b_{k+1}$. If these both have order 2 then $G$ is the homomorphic image of a dihedral group, so we shall assume that only one has order 2.

Let $b_{k+1}^{2}=1$. If one of $b_{j+1}$ and $b_{2}$ has order 3 and the other one has order less than 6 then $G$ is the homomorphic image of a finite triangle group, so we shall assume that this is not the case. In all the remaining cases it is possible to allocate weights that avoid critical cycles.

The case where $b_{k+1}^{2} \neq 1$ is dealt with in essentially the same way, so we omit the details.

Lemma 5.7. Let $b_{i}^{2}=1$ for some $i$. Then $\Sigma$ has a solution over $G$.

Proof. On account of Corollary 5.4, Lemma 5.5 and Lemma 5.6 we may assume that $c_{1} \ldots c_{n}=1$, not all $b_{i}$ have order 2 and 


$$
a_{1} c_{1} \ldots c_{j-1} a_{j}^{-1} b_{j+1}^{\eta} a_{j} c_{j} \ldots c_{k-1} a_{k}^{-1} b_{k+1}^{\epsilon} a_{k} c_{k} \ldots c_{n} a_{1}^{-1} b_{2}^{\delta} \neq 1
$$

where $\eta, \epsilon, \delta \in\{ \pm 1\}$. As in the proof of Lemma 5.6 it is sufficient to study the case where $b_{2}^{2} \neq 1$ and we begin by choosing the following weights:

$$
\begin{gathered}
\omega\left(a_{i}\right)=\left\{\begin{array}{cl}
1 / 3 & \text { for } 1<i<j \\
-1 / 3 & \text { for } i \in\{1, j\} \\
-1 & \text { for } i=k \\
0 & \text { else }
\end{array}\right. \\
\omega\left(b_{i}\right)= \begin{cases}2 / 3 & \text { for } 1<i \leq j \\
1 & \text { else }\end{cases} \\
\omega\left(c_{i}\right)= \begin{cases}1 & \text { for } i=k \\
2 / 3 & \text { for } i=j \\
1 / 3 & \text { for } i=1 \\
0 & \text { else }\end{cases}
\end{gathered}
$$

The critical cycles which will de dealt with individually are:

$$
\begin{aligned}
& a_{j} c_{j} \ldots c_{k-1} a_{k}^{-1} b_{k+1} a_{k} c_{k-1}^{-1} \ldots c_{j}^{-1} a_{j}^{-1} b_{j+1}^{ \pm 2} \\
& a_{j} c_{j} \ldots c_{k-1} a_{k}^{-1} b_{k+1}^{ \pm 2} a_{k} c_{k-1}^{-1} \ldots c_{j}^{-1} a_{j}^{-1} b_{j+1} \\
& a_{1} c_{1} \ldots c_{j-1} a_{j}^{-1} b_{j+1} a_{j} c_{j-1}^{-1} \ldots c_{1}^{-1} a_{1}^{-1} b_{2}^{ \pm 2} \\
& a_{k} c_{k} \ldots c_{n} a_{1}^{-1} b_{2}^{ \pm 2} a_{1} c_{n}^{-1} \ldots c_{k}^{-1} a_{k}^{-1} b_{k+1}
\end{aligned}
$$

and the ones induced by

$$
a_{j} c_{j} \ldots c_{k-1} a_{k}^{-1} b_{k+1} a_{k} c_{k-1}^{-1} \ldots c_{j}^{-1} a_{j}^{-1} b_{j+1} .
$$

There are in fact more critical cycles than these; for instance

$$
a_{j} c_{j} \ldots c_{k-1} a_{k}^{-1} b_{k+1} a_{k} c_{k-1}^{-1} \ldots c_{j}^{-1} a_{j}^{-1} b_{j+1}^{ \pm 2}
$$

is a relator if and only if

$$
a_{i} c_{i} \ldots c_{k-1} a_{k}^{-1} b_{k+1} a_{k} c_{k-1}^{-1} \ldots c_{i}^{-1} a_{i}^{-1} b_{i+1}^{ \pm 2} \text { for } j<i<k
$$


is, but we do not list them since they can all be dealt with in one go. Hence there are five types of cycles to be studied, but since the first four are similar we only give the details of (4). We then assume that none of (4), (5), (6) and (7) are admissible and show how to deal with the cycles induced by (8).

Let $a_{j} c_{j} \ldots c_{k-1} a_{k}^{-1} b_{k+1} a_{k} c_{k-1}^{-1} \ldots c_{j}^{-1} a_{j}^{-1} b_{j+1}^{ \pm 2}=1$. Then we may assume that cycles with label $a_{1} c_{1} \ldots c_{j-1} a_{j}^{-1} b_{j+1} a_{j} c_{j-1}^{-1} \ldots c_{1}^{-1} a_{1}^{-1} b_{2}^{ \pm m}$ are not admissible as they would make $G$ cyclic. Since $b_{k+1} b_{j+1}^{ \pm 2}=1$ it follows that $b_{j+1}^{2} \neq 1$, so $b_{k+1}^{2}=1$ and hence $b_{j+1}$ has order 4 . This means that $b_{j+1}^{2} b_{2}$ cannot be a relator since this would imply $b_{2}^{2}=1$, a contradiction.

Let $b_{2}$ have order at least 4 and choose the following weights:

$$
\begin{gathered}
\omega\left(a_{i}\right)=\left\{\begin{aligned}
1 / 2 & \text { for } 1<i<j \\
-1 / 2 & \text { for } i \in\{1, k\} \\
0 & \text { else }
\end{aligned}\right. \\
\omega\left(b_{i}\right)= \begin{cases}1 / 2 & \text { for } 1<i \leq k \\
1 & \text { else }\end{cases} \\
\omega\left(c_{i}\right)= \begin{cases}1 & \text { for } i=k \\
1 / 2 & \text { for } i=1 \text { or } j \leq i<k \\
0 & \text { else }\end{cases}
\end{gathered}
$$

In this case there are no critical cycles.

Now let $b_{2}$ have order 3. The fact that $a_{k} c_{k} \ldots c_{n} a_{1}^{-1} b_{2}^{ \pm 2} a_{1} c_{n}^{-1} \ldots c_{k}^{-1} a_{k}^{-1} b_{k+1}=1$ if and only if $a_{i} c_{i} \ldots c_{n} a_{1}^{-1} b_{2}^{ \pm 2} a_{1} c_{n}^{-1} \ldots c_{i}^{-1} a_{i}^{-1} b_{i+1}=1$ for $k<i<n$ means that we have to consider two cases here, one for $k=n$ and one for $k<n$. If $k=n$ we allocate weights as shown below:

$$
\begin{aligned}
& \omega\left(a_{i}\right)=\left\{\begin{aligned}
1 / 2 & \text { for } j<i<k \\
1 / 3 & \text { for } 1<i<j \\
-1 / 3 & \text { for } i=k \\
-5 / 6 & \text { for } i=1 \\
0 & \text { else }
\end{aligned}\right. \\
& \omega\left(b_{i}\right)= \begin{cases}2 / 3 & \text { for } 1<i \leq j \\
1 / 2 & \text { for } j<i \leq k \\
1 & \text { else }\end{cases} \\
& \omega\left(c_{i}\right)= \begin{cases}5 / 6 & \text { for } i \in\{1, k\} \\
1 / 3 & \text { for } i=j \\
0 & \text { else }\end{cases}
\end{aligned}
$$

The only critical cycle has label $a_{n} c_{n} a_{1}^{-1} b_{2}^{ \pm 2} a_{1} c_{n}^{-1} a_{n}^{-1} b_{1}$, but if this is an admissible path 
then $1=b_{2}^{ \pm 2} b_{k+1}$, so $b_{2}$ has order 4 , a contradiction.

If $k<n$

$$
\begin{aligned}
& \omega\left(a_{i}\right)=\left\{\begin{aligned}
1 / 3 & \text { for } 1<i<j \\
-1 / 3 & \text { for } i \in\{1, k\} \\
-1 / 2 & \text { for } i=k+1 \\
0 & \text { else }
\end{aligned}\right. \\
& \omega\left(b_{i}\right)= \begin{cases}2 / 3 & \text { for } 1<i \leq j \\
1 / 2 & \text { for } j<i \leq k \\
1 & \text { else }\end{cases} \\
& \omega\left(c_{i}\right)= \begin{cases}5 / 6 & \text { for } i=k \\
1 / 2 & \text { for } i=k+1 \\
1 / 3 & \text { for } i \in\{1, j\} \\
0 & \text { else }\end{cases}
\end{aligned}
$$

The cycles with label $a_{i} c_{i} \ldots c_{n} a_{1}^{-1} b_{2}^{ \pm 2} a_{1} c_{n}^{-1} \ldots c_{i}^{-1} a_{i}^{-1} b_{i+1}$ where $k<i \leq n$ are the critical ones, and the same argument holds as in the case where $k=n$.

From now on we shall assume that none of $b_{k+1} b_{j+1}^{ \pm 2}, b_{k+1} b_{2}^{ \pm 2}, b_{j+1} b_{k+1}^{ \pm 2}$ and $b_{j+1} b_{2}^{ \pm 2}$ is a relator in $G$, and the only cases we need to study to complete the proof of this lemma are the critical cycles by (8).

We begin with the easier cases where $b_{j+1}$ and $b_{k+1}$ have order greater than 2 . If $b_{j+1}$ has order greater than 2 we choose the following weights:

$$
\begin{aligned}
& \omega\left(a_{i}\right)=\left\{\begin{array}{cl}
1 / 3 & \text { for } 1<i<j \\
-2 / 3 & \text { for } i \in\{1, k\} \\
0 & \text { else }
\end{array}\right. \\
& \omega\left(b_{i}\right)= \begin{cases}2 / 3 & \text { for } 1<i \leq k \\
1 & \text { else }\end{cases} \\
& \omega\left(c_{i}\right)= \begin{cases}1 & \text { for } i=k \\
2 / 3 & \text { for } i=1 \\
1 / 3 & \text { for } j \leq i<k \\
0 & \text { else }\end{cases}
\end{aligned}
$$

and in this case there are no critical cycles, so let us now assume that $b_{j+1}$ has order 2.

If $b_{k+1}$ has order greater than 2 we choose 


$$
\begin{gathered}
\omega\left(a_{i}\right)=\left\{\begin{array}{cl}
0 & \text { for } 1 \leq i<j \\
-2 / 3 & \text { for } i \in\{j, k\} \\
1 / 3 & \text { else }
\end{array}\right. \\
\omega\left(b_{i}\right)= \begin{cases}1 & \text { for } i=k \\
2 / 3 & \text { else }\end{cases} \\
\omega\left(c_{i}\right)= \begin{cases}1 & \text { for } i=j \\
2 / 3 & \text { for } i=k \\
1 / 3 & \text { for } 1 \leq i<j \\
0 & \text { else }\end{cases}
\end{gathered}
$$

provided $k=j+1$, and if $k>j+1$ we choose

$$
\begin{gathered}
\omega\left(a_{i}\right)=\left\{\begin{aligned}
1 / 3 & \text { for } k<i \leq n \\
-2 / 3 & \text { for } i=j \\
-1 / 3 & \text { for } i \in\{j+1, k\} \\
0 & \text { else }
\end{aligned}\right. \\
\omega\left(b_{i}\right)= \begin{cases}1 & \text { for } j<i \leq k \\
2 / 3 & \text { else }\end{cases} \\
\omega\left(c_{i}\right)= \begin{cases}1 & \text { for } i=j \\
1 / 3 & \text { for } 1 \leq i<j \text { and } i \in\{j+1, k\} ; \\
0 & \text { else }\end{cases}
\end{gathered}
$$

again there are no critical cycles, so we may assume that $b_{k+1}$ has order 2 too. Hence the only critical relator induced by

$$
a_{j} c_{j} \ldots c_{k-1} a_{k}^{-1} b_{k+1} a_{k} c_{k-1}^{-1} \ldots c_{j}^{-1} a_{j}^{-1} b_{j+1}^{ \pm 1}
$$

is $\left(b_{k+1} b_{j+1}\right)^{2}$.

Let $b_{2}$ have order greater than 3 . In this case we choose the following weights:

If $k=j+1$

$$
\begin{aligned}
& \omega\left(a_{i}\right)=\left\{\begin{array}{cl}
1 / 2 & \text { for } 1<i<j \\
-1 / 4 & \text { for } i \in\{1, j\} \\
-1 & \text { for } i=k \\
0 & \text { else }
\end{array}\right. \\
& \omega\left(b_{i}\right)= \begin{cases}1 / 2 & \text { for } 1<i \leq j \\
1 & \text { else }\end{cases}
\end{aligned}
$$




$$
\omega\left(c_{i}\right)= \begin{cases}1 & \text { for } i=k \\ 3 / 4 & \text { for } i=j \\ 1 / 4 & \text { for } i=1 \\ 0 & \text { else }\end{cases}
$$

if $k>j+1$

$$
\begin{gathered}
\omega\left(a_{i}\right)=\left\{\begin{array}{cl}
1 / 2 & \text { for } 1<i<j \\
-1 / 4 & \text { for } i \in\{1, j\} \\
-1 / 2 & \text { for } i \in\{j+1, k\} \\
0 & \text { else }
\end{array}\right. \\
\omega\left(b_{i}\right)= \begin{cases}1 / 2 & \text { for } 1<i \leq j \\
1 & \text { else }\end{cases} \\
\omega\left(c_{i}\right)= \begin{cases}3 / 4 & \text { for } i=j \\
1 / 2 & \text { for } i \in\{j+1, k\} \\
1 / 4 & \text { for } i=1 \\
0 & \text { else }\end{cases}
\end{gathered}
$$

in either case there are no critical cycles.

Now let $b_{2}^{3}=1$ and choose weights as shown below:

If $k=j+1$

$$
\begin{aligned}
& \omega\left(a_{i}\right)=\left\{\begin{array}{cl}
-1 / 3 & \text { for } i=1 \\
-1 / 2 & \text { for } i=j \\
-5 / 6 & \text { for } i=k \\
1 / 3 & \text { for } 1<i<j \\
0 & \text { else }
\end{array}\right. \\
& \omega\left(b_{i}\right)= \begin{cases}2 / 3 & \text { for } 1<i \leq j \\
1 & \text { else }\end{cases} \\
& \omega\left(c_{i}\right)= \begin{cases}5 / 6 & \text { for } i \in\{j, k\} \\
1 / 3 & \text { for } i=1 \\
0 & \text { else }\end{cases}
\end{aligned}
$$

If $k>j+1$

$$
\omega\left(a_{i}\right)=\left\{\begin{array}{cl}
-1 / 3 & \text { for } i \in\{1, j+1\} \\
-1 / 2 & \text { for } i \in\{j, k\} \\
1 / 3 & \text { for } 1<i<j \\
0 & \text { else }
\end{array}\right.
$$




$$
\begin{gathered}
\omega\left(b_{i}\right)= \begin{cases}2 / 3 & \text { for } 1<i \leq j \\
1 & \text { else }\end{cases} \\
\omega\left(c_{i}\right)= \begin{cases}5 / 6 & \text { for } i=j \\
1 / 3 & \text { for } i \in\{1, j+1\} . \\
0 & \text { else }\end{cases}
\end{gathered}
$$

The critical cycles are induced by $a_{1} c_{1} \ldots c_{j-1} a_{j}^{-1} b_{j+1} a_{j} c_{j-1}^{-1} \ldots c_{1}^{-1} a_{1}^{-1} b_{2}$, so the corresponding relators are $\left(b_{j+1} b_{2}\right)^{2}$ and $\left[b_{j+1}, b_{2}\right]$. If either of these is a relator then we choose the following weights:

$$
\begin{gathered}
\omega\left(a_{i}\right)= \begin{cases}-1 / 2 & \text { for } i=1 \\
-1 / 3 & \text { for } i=j \\
-5 / 6 & \text { for } i=k \\
1 / 3 & \text { for } 1<i<j \\
0 & \text { else }\end{cases} \\
\omega\left(b_{i}\right)= \begin{cases}2 / 3 & \text { for } 1<i \leq j \\
1 & \text { else }\end{cases} \\
\omega\left(c_{i}\right)= \begin{cases}5 / 6 & \text { for } i=k \\
2 / 3 & \text { for } i=j \\
1 / 2 & \text { for } i=1 \\
0 & \text { else }\end{cases}
\end{gathered}
$$

Now the critical cycles are induced by $a_{1} c_{n}^{-1} \ldots c_{k}^{-1} a_{k}^{-1} b_{k+1} a_{k} c_{k} \ldots c_{n} a_{1}^{-1} b_{2}$ and these are the relators $\left(b_{k+1} b_{2}\right)^{2}$ and $\left[b_{k+1}, b_{2}\right]$; in all these cases $G$ is a finite group.

This completes the final section of this paper, and the following lemma summarises our result:

Lemma 5.8. If $|K|=3$, then $\Sigma$ has a solution over $G$.

Proof. This follows from Lemma 5.1 and Lemma 5.7.

We have now proved our theorem by showing that $\Sigma$ has a solution for any value of $K$.

\section{REFERENCES}

1. W. A. Bogley and S. J. PRIDE, Aspherical relative presentations, Proc. Edinburgh Math. Soc. 35 (1992), 1-39.

2. M. D. E. ConDER, Three-relator quotients of the modular group, Quart. J. Math. Oxford (2) 38 (1987), 427-447. 
3. S. M. Gersten, Reducible diagrams and equations over groups, in Essays in group theory (MSRI Publications 8, edited by S. M. Gersten, Springer-Verlag, 1987).

4. M. Gerstenhaber and O. S. Rothaus, The solution of equations in groups, Proc. Nat. Acad. Sci. USA 48 (1962), 1531-1533.

5. J. HowIE, On pairs of 2-complexes and systems of equations in groups, J. Reine Angew. Math. 324 (1981), 165-174.

6. J. Howre, The solution of length three equations over groups, Proc. Edinburgh Math. Soc. 26 (1983), 89-96.

7. J. HowIE, Nonsingular systems of two length three equations over a group, Math. Proc. Cambridge Philos. Soc. (11), 110 (1991), 11-24.

8. V. Metaftsis, Studies on one-relator products of groups (Ph.D. thesis, Heriot-Watt University, 1994).

9. O. S. Rothaus, On the non-triviality of some group extensions given by generators and relations, Ann. of Math. (2) 106 (1977), 559-612.

10. MARTin SchöNert et al. GAP - Groups, Algorithms, and Programming (Lehrstuhl D. für Mathematik, Rheinisch Westfälische Technische Hochschule, Aachen, Germany, third edition, 1993).

11. S. WRETH, Hyperbolicity of one-relator products and equations over groups (Ph.D. thesis, Heriot-Watt University, 1995).

Department of Mathematics

HERIOT-WATT UNIVERSITY

RICCARTON

EDINBURGH EH14 4AS 